\title{
Antioxidant ofsmallmolecular weightchitosan oligosaccharidein vitro
}

\author{
Shang Kong ${ }^{\#}$, Peiqiu Cao\#, Jiao Guo and Zhengquan $\mathrm{Su}^{\mathrm{a}}$ \\ Key Research Center of Liver Regulation for Hyperlipidemia SATCM/Class III Laboratory of Metabolism SATCM, \\ Guangdong TCM Key Laboratory for Metabolic Diseases, Guangdong Pharmaceutical University, Guangzhou, \\ 510006, China
}

\begin{abstract}
In recent years, there has been increasing interest in finding natural antioxidants, since they can protect the human body from free radicals and retard the progress of many chronic diseases. This study is to evaluate antioxidant of a certain molecular chitosan oligosaccharide (Mw $1000 \mathrm{Da}, \mathrm{COST})$ in vitro. The antioxidant activities of COST were investigated in vitro by several antioxidant assays, including DPPH radical scavenging, hydroxyl radical scavenging, superoxide radical scavenging, reducing power and metal chelating activities.In vitro studies found that COST had noticeable scavenging activities on 2,2diphenyl-1-picryl-hydrazyl (DPPH) radical, hydroxyl radical, and superoxide anion, and possessed reducing power and metal chelating activities, and its capability of anti-oxidation enhance with the increase of concentration.COST presents anexcellent biological rolein scavenging free radical, which could be explored as a potential antioxidant or dietary supplement to retard diseases caused by peroxide.
\end{abstract}

\section{Introduction}

The increase of mean life in the industrialized world will contribute to $5 \%$ of the population older than $85 \mathrm{yr}$ of agein $2050[1]$.With the research about the molecular mechanism of aging, the 'free radical theory of ageing' is showing promise in helping to understand the process of ageing and in finding effective anti-ageing agents[2]. The theory postulates that aging is caused by excessive reaction of free radicals in living things, which leads to cell death and tissue injury by causing cellular damage[3]. Excessive accumulation of ROS increases the chances of the onset of diseases, indeed, it is linked to biological wear and tear leading to certain types of age-related diseases including cardiovascular disease[4], Type II diabetes[5], the decline of female reproductive function[6] and so on. So people have serious implications with respect to interventional strategies scavenging free radical to delay senescence. Chitosan oligosaccharides (COS), as degradation product of chitosan (CTS), have been

\footnotetext{
${ }^{a}$ Corresponding author. E-Mails: suzhq@scnu.edu.cn (Z.-Q.S.) and wshxalb@163.com (J.G.).

"Shang Kong and Peiqiu Cao make equal contributions to this work.

This project was financially supported by the National Science Foundation of China (no. 81173107), the Science and Technology Planning Project of Guangdong, China(2013B021100018).
} 
extensively applied in pharmacy, food and other fields becauseof good biocompatibility, degradation, non-toxicity and easy-absorbability[7].Several studies have demonstrated that COS and related products have beneficial effects on weight loss[8] and anti-hyperlipidemia[9]. In recent years, some studies have shown that COS had obviously inhibitory effects on oxidation and glycation, its activities were linked with degrees of deacetylation (DD)[10]. Furthermore different molecular weight of COS was likely to contribute significantly towards the antioxidant effect, there was inversely proportional relationship between them $[11,12]$. But there are currently no found one research that evaluates antioxidant activities a certain molecular chitosan oligosaccharide (Mw $1000 \mathrm{Da}, \mathrm{DD} \geq 91 \%$, COST) in vitro.In vitro the antioxidant activities of COST were investigated in vitro by several antioxidant assays, including hydroxyl radical scavenging, superoxide radical scavenging, reducing power and metal chelating activities.

\section{Materials and methods}

\subsection{Materials}

COST (Mw1000 Da; DD 95.6\%) was purchased from Shandong Aokang Biotech Ltd., Shandong, China. 2,2-diphenyl-1-picryl-hydrazyl (DPPH) was obtained from Shanghai Mai Kelin Biochemical Technology Ltd., Shanghai, China. Nitro blue tetrazolium (NBT), phenazinemethosulfate (PMS), nicotinamide adenine dinucleotide-reduced (NADH) were purchased from Shanghai Aladdin Reagent Ltd., Shanghai, China. All other reagents and solvents were of analytical grade.

\subsection{In vitro antioxidant activities of COST}

\subsubsection{DPPH assay}

Free radical scavenging ability was determined by testingthe change of the absorption by $517 \mathrm{~nm}$ before and after the reaction of DPPH and antioxidant substances.COST samples was dissolved in 1 $\mathrm{mL}$ of distilled water at the different concentrations taken in test tubesof Sample group and was added $1.0 \mathrm{ml}$ of $0.1 \mathrm{mMethanol} \mathrm{solution}$ of DPPH, mixed well and allowed to incubate at room temperature for $30 \mathrm{~min}$ in the dark. At the same time, one test tubes of Control group was mixed with $1.0 \mathrm{~mL}$ DPPH and $1.0 \mathrm{~mL}$ distilled water, and the sample solution of Blank group was mixed with $1.0 \mathrm{~mL}$ COST and $1.0 \mathrm{~mL}$ anhydrous alcohol. The absorbance value of the sample was measured at $517 \mathrm{~nm}$. The percentage of DPPH radical scavenging activity was theoretically calculated by the following formula:

$$
\text { DPPH radical scavenging activity }(\%)=\left(1-\frac{A_{\text {sample }}-A_{\text {blenk }}}{A_{\text {contral }}}\right) \times 100 \%
$$

\subsubsection{Hydroxyl radical scavenging assay}

The hydroxyl radical scavenging ability of COST was measured according to a method described previously with some modification[13]. Briefly, the different concentration of COST was mixedwith $1.0 \mathrm{~mL} \mathrm{FeSO}_{4}(6.0 \mathrm{mM}), 1.0 \mathrm{~mL} \mathrm{H}_{2} \mathrm{O}_{2}(2.5 \mu \mathrm{M})$, respectively. The mixture was then incubated at room temperature for $10 \mathrm{~min}$. The reaction was initiated upon addition of $1.0 \mathrm{~mL}$ salicylic acid solution $(6.0 \mathrm{mM})$. The absorbance was determined at $500 \mathrm{~nm}$ after $30 \mathrm{mins}$ reaction time at $37^{\circ} \mathrm{C}$. The ability of hydroxyl radical scavenging ( $\%$ ) was calculated as follows:

$$
\text { Scavenging rate }(\%)=\frac{A_{0}-\left(A_{1}-A_{2}\right)}{A_{0}} \times 100 \%
$$

Where $A_{1}$ was the absorbance of the sample and $A_{0}$ was the absorbance of the solvent control, whereas 
$\mathrm{A}_{2}$ was the absorbance of the reagent solution without sodium salicylate.

\subsubsection{Superoxide anion radical scavenging assay}

The scavenging assay of superoxide anion radical was carried out according to the method with a little modification. COST samples with different concentrations were prepared with distilled water. The reaction mixture containing of $1.0 \mathrm{~mL}$ of test samples, $1.0 \mathrm{~mL}$ NADH $(338 \mu \mathrm{M}), 1.0 \mathrm{~mL}$ NBT $(72 \mu \mathrm{M})$ and $1.0 \mathrm{~mL}$ PMS $(300 \mu \mathrm{M})$ was reacted at room temperature for $5 \mathrm{~min}$. Absorbance of the resulting solution was measured at $560 \mathrm{~nm}$ on a spectrophotometer against a blank. The scavenging ability of superoxide anion radical was calculated by the following equation:

$$
\text { Scavenging activity }(\%)=1-\frac{A}{A_{0}} \times 100 \%
$$

Where Asample was the absorbance of sample group and $\mathrm{A}_{0}$ was the absorbance of blank control group (Tris-HCl buffer instead of NADH).

\subsubsection{Measurement of reducing power}

The experiment was performed by the modified method. Briefly, $1 \mathrm{ml}$ COST with different concentrations were added to $1.0 \mathrm{ml}$ of potassium ferricyanide $(1 \% \mathrm{w} / \mathrm{v})$ and $0.2 \mathrm{~mL}$ phosphate buffer (0.2 M pH6.6), respectively. The mixed solution was shaken well and incubated at $50^{\circ} \mathrm{C}$ for $20 \mathrm{~min}$. Then $1.0 \mathrm{ml}$ of trichloroacetic acid solution $(10 \% \mathrm{w} / \mathrm{v})$ was added to the mixture with the purpose of terminating the reaction. After $6000 \mathrm{r} / \mathrm{min}$ centrifuge for $10 \mathrm{~min}, 0.35 \mathrm{ml}$ of $3 \%$ ferric chloride was added to $1 \mathrm{ml}$ supernatant of mixture. Absorbance of final reaction product was read at $700 \mathrm{~nm}$ on a spectrophotometer against a blank after $15 \mathrm{~min}$ at room temperature.

\subsubsection{Metal chelating activity(11)}

The different concentrations of COST samples were added to a mixture of $0.05 \mathrm{ml} \mathrm{FeCl} 2(2 \mathrm{mM})$ and $1 \mathrm{ml}$ deionized water, then stewing $2 \mathrm{~min}$. The reaction was initiated by the addition of 5 mMferrozine $(1 \mathrm{~mL})$. After the mixture had reached equilibrium, the absorbance of the solution was then measured spectrophotometrically at $562 \mathrm{~nm}$. The ability of COST to chelate ferrous ion was calculated using the following equation:

$$
\text { Chelating ability }(\%)=\frac{A_{2}-\left(A_{0}-A_{1}\right)}{A_{2}} \times 100 \%
$$

where A0 and A2 were the optical density at $562 \mathrm{~nm}$ without and with samples, respectively. A1 was the absorbance of the group without $\mathrm{FeSO}_{4}$

\section{Results}

\subsection{DPPH scavenging activity}

DPPH is a free radical which has unpaired electron in its middle nitrogen atom. DPPH can pairs with a free radical scavenger to yield a colored change gradually[13]. Therefore, antioxidative ability of COST can be evaluated by the assay of scavenging DPPH radical. The scavenging effects of COST on DPPH radical were shown in (Figure 1) exhibited COST has obvious scavenging effect to DPPH, and the scavenging activity is growing with increasing the concentration. The active amino and hydroxyl groups in COST molecules canprovide the hydrogen ions to bind with DHHP, which can achieve the purpose of removing the DPPH free radical. 


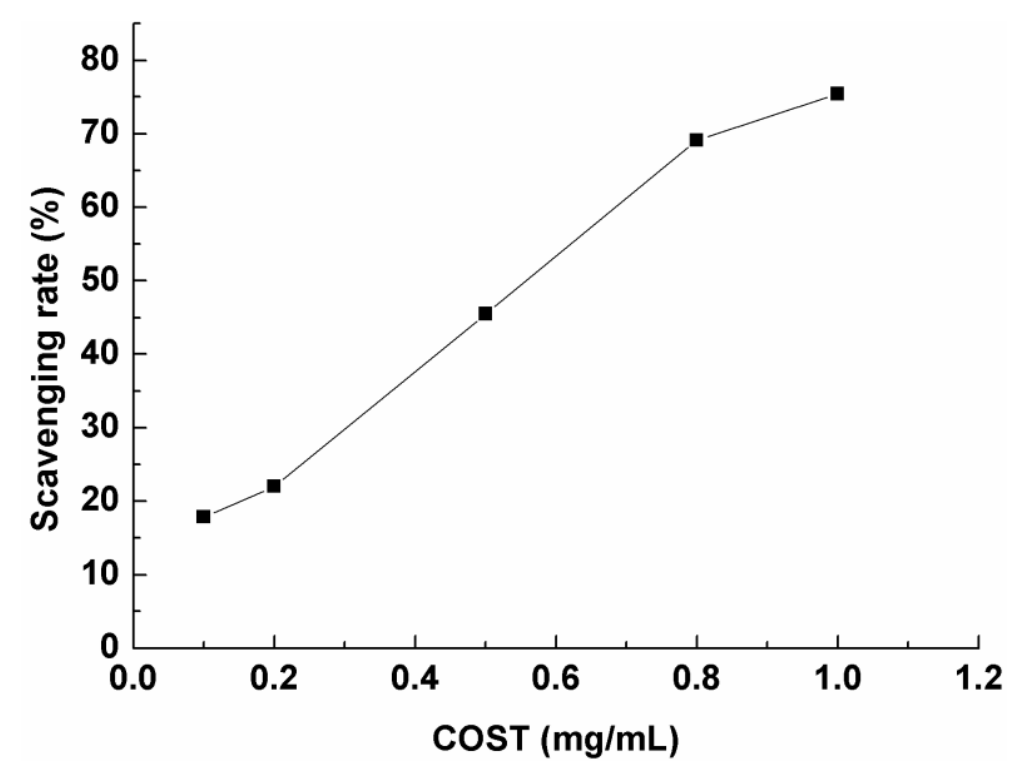

Figure 1. Scavenging effect COST with different concentrations towards DPPH radicals.

\subsection{Hydroxyl free radical $(\cdot \mathrm{OH})$ scavenging activity}

Hydroxyl radical is one of the most reactive and dangerous free radicals among the reactive oxygen species, which can cause serious damage to the adjacent biological molecules by reaction of Fe (II) complex with $\mathrm{H}_{2} \mathrm{O}_{2}$ in the presence of salicylic acid[14]. In this study, we evaluated the scavenging activity of COST on $\mathrm{HO} \cdot$ generated through the Fenton reaction. As shown in Figure 2, the scavenging capacities of COST were elevated quickly with theincrease of concentration. Fenton reaction is one of the important mechanisms in $\cdot \mathrm{OH}$. The removal rate of $\cdot \mathrm{OH}$ is an important indicator of antioxidant capacity[15]. The results of this experiment can be used as evidence of the antioxidant capacity of COST, and provide clues for mechanism of its antioxidant in the further study.

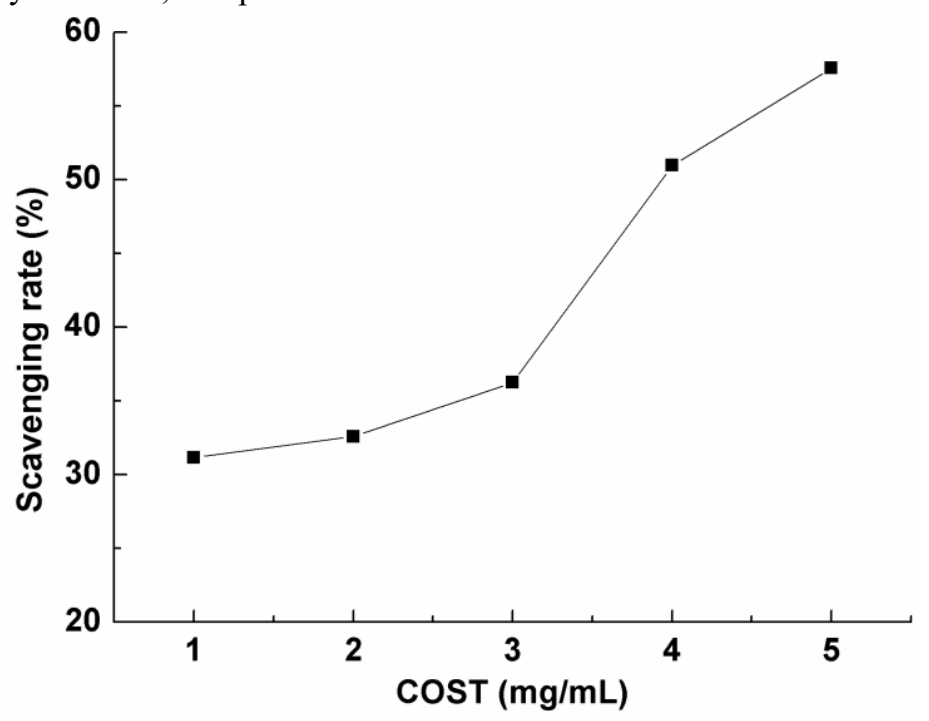

Figure 2. Scavenging effect of COST with different concentrations towards hydroxyl radicals 


\subsection{Superoxide anion radical scavenging activity}

Compare to other radicals, superoxide anionradical is a relatively weak oxidant in most organisms, but it has much longer lifetime and could form secondary radicals such as hydrogen peroxide and hydroxylradicalthat causetissue damages and various diseases[16]. Therefore, the scavenging effect on superoxide radical is an important way to illustrate the mechanism of antioxidant activity. In Figure 3, we found that when the concentration of COST was less than $0.5 \mathrm{mg} / \mathrm{ml}$, the scavenging activity is weak. Once the concentration of COST was more than $0.5 \mathrm{mg} / \mathrm{ml}$, the scavenging effects of COST at various concentrations on superoxide radical were dose-dependent. Superoxide radical is the first oxygen free radicals formed by ground state oxygen received an electron, and may be other radicals formed by a series of reactions. COST can react with oxygen free radicals, so that oxygen radicals will be completely removed. The result shows that COST has rather highability of scavenging superoxide radical.

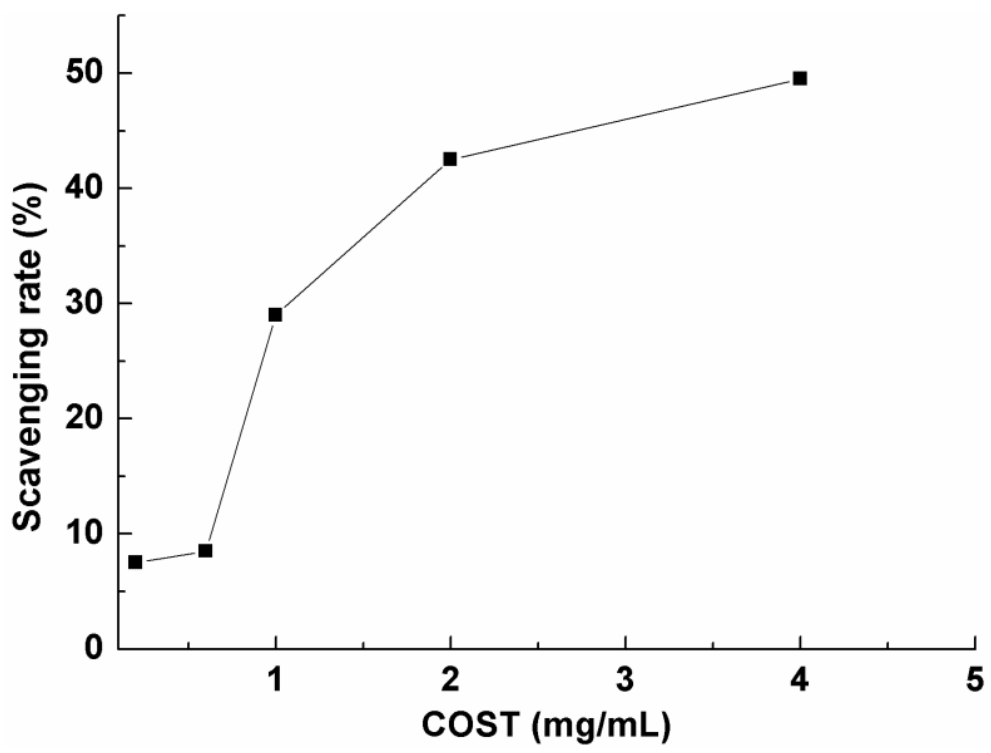

Figure 3. Scavenging effect of COST with different concentrations towards superoxide radicals.

\subsection{Reducing power}

Usually reductant provides electron to reduce free radicals produced by oxidative stress. Antioxidant reduces $\mathrm{Fe}^{3+}$ to $\mathrm{Fe}^{2+}$ under a certain condition and absorbance ofreaction mixture is read at $700 \mathrm{~nm}$ [17]. Higher absorbance of antioxidant indicates it has greater reducing power. The reducing powers of COST were shown in Figure 4, reducing power increased with increasing concentration of COST. The reducing properties are generally associated with the presence of reductones, which have been shown to exert antioxidant action by breaking the free radical chain by donating a hydrogen atom[11].Reductones are also reported to react with certain precursors of peroxide, thus preventing peroxide formation. Our result indicates it was likely to contribute significantly towards the observed potential antioxidant activity. 


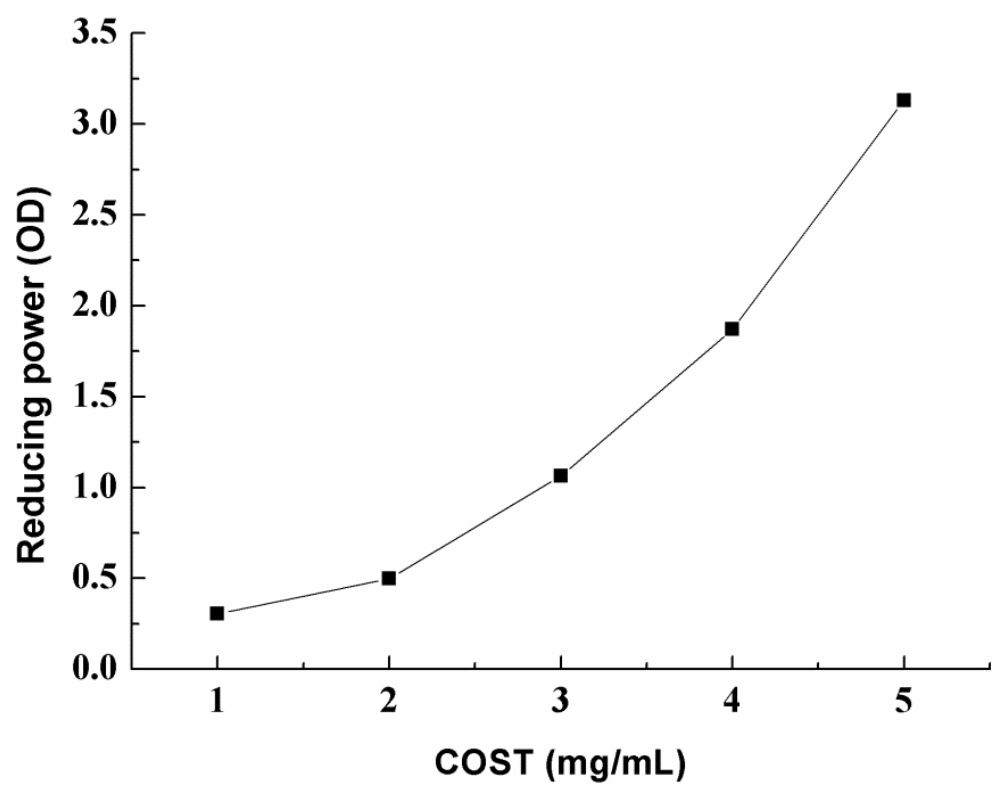

Figure 4. Reducing power of COST with different concentrations

\subsection{Chelating ability}

Ferrous ion chelating ability is another important indicator to evaluate an antioxidant's capacity in vitro. The chelating ability of COST against $\mathrm{Fe}^{2+}$ is shown in Figure 5 that the chelating ability were elevated quickly with the increase of concentration of COST. $\mathrm{Fe}^{2+}$ was thought to be one encouragement of causing oxygen free radicals and promoting oil peroxide, it can prevents oxidative damage by reducing the concentration of $\mathrm{Fe}^{2+}$ generated in the Fenton reaction[18]. Our dates demonstrate that the form of COST and $\mathrm{Fe}^{2+}$ complex can greatly improve the stability of $\mathrm{Fe}^{2+}$, which may contribute to the prevention of cardiovascular disease.

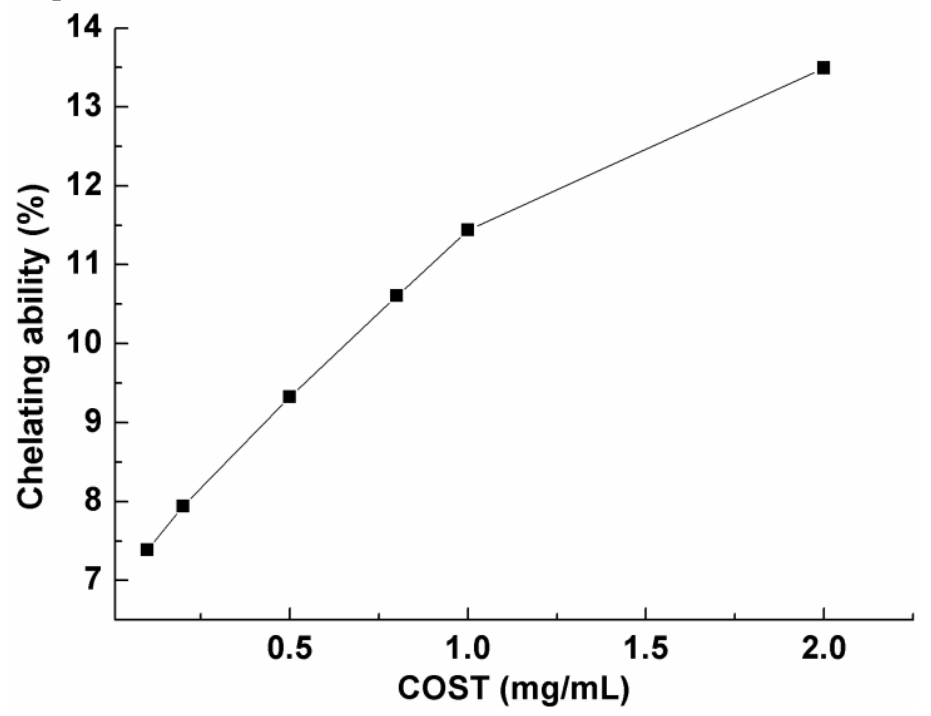

Figure 5. Chelating effect of COST with different concentrations on ferrous ions 


\section{Discussion and Conclusion}

In normal condition, Aging was usually known asa process that when the biological development is mature, with the increase of age, its own physiological functionwill decrease accompanying with the decline in the stability of internal environment, and the structure, group will gradually degenerated in change, leading to die, which is anirreversible phenomenon[19]. Once excessive accumulation of free radicals such as superoxide anion radical, hydroxyl radical and so on in the body, that results a series of free radical reaction, which accelerate the aging process resulting in high incidence of hyperlipidemia, hypertension, diabetes, Alzheimer's disease, Parkinson's disease and cancer [20-22].It is thus rapidly becoming an urgent necessity to develop safe and effective types of antioxidants that may scavenge free radical todelay senescence.

Chitosan (CTS) is a polysaccharide material extracted from shell; it is generally considered a good excipient because of its biocompatibility and show a variety of activities such as antihyperlipidemia[23], lose weight[24], antioxidant[25]. As the small molecular derivatives of CTS, COS have shown the better water-solubility and thus can be digested and absorbed more easily by the gastrointestinal tract in animals and humans, which may make them better biological activity[26-28]. COST, one type of COS, is known byits certain molecular weight $1000 \mathrm{Da}$, which may possess more significant biological activity of antioxidant.

In this study, we firstly explore its antioxidant function in intro.Reactive oxygen species (ROS), including superoxide anion radicals, hydroxyl radicals, and hydrogen peroxide etc., are often generated by oxidation product of biological reactions or exogenous factors. The study of its antioxidant activity in vitro, has shown that COST can scavenge DPPH radical, hydroxyl radical, and superoxide anion, and possess reducing power and metal chelating activities, which expressed high antioxidant activity and the activity depended on its molecular weight and concentration.

Excessive generation of ROS, induced by various stimulating factors such as certain pollutants, tobacco smoke, and which exceed the antioxidant capacity of the organism will lead to aging, cancer, and other many diseases. The results of experiment in vitro was given to us that COST may play an important role in the elimination of ROS and protect the cells against toxic effects of ROS.

\section{References}

1. Tuljapurkar, S., Li, N., and Boe, C., A universal pattern of mortality decline in the G7 countries, Nature, 405, 789 (2000).

2. Harman, D., Free-radical theory of aging. Increasing the functional life span, Ann NYAcadSci, 717, 1 (1994).

3. Ho, Y. S., Magnenat, J. L., Gargano, M., and Cao, J., The nature of antioxidant defense mechanisms: a lesson from transgenic studies, Environ Health Perspect, 106 Suppl5, 1219 (1998).

4. Cao, P., Pan, H., Xiao, T., Zhou, T., Guo, J., and Su, Z., Advances in the Study of the Antiatherogenic Function and Novel Therapies for HDL, Int J MolSci, 16, 17245 (2015).

5. Masoro, E. J., Physiology of aging, Int J Sport NutrExercMetab, 11Suppl, S218 (2001).

6. Tatone, C., Di Emidio, G., Vitti, M., Di Carlo, M., Santini, S. J., D'Alessandro, A. M., Falone, S., and Amicarelli, F., Sirtuin Functions in Female Fertility: Possible Role in Oxidative Stress and Aging, Oxid Med Cell Longev, 2015, 659687 (2015).

7. Hossain, S., Rahman, A., Kabir, Y., Shams, A. A., Afros, F., and Hashimoto, M., Effects of shrimp (Macrobraciumrosenbergii)-derived chitosan on plasma lipid profile and liver lipid peroxide levels in normo- and hypercholesterolaemic rats, ClinExpPharmacolPhysiol, 34, 170 (2007).

8. Cao, P., Huang, G., Yang, Q., Guo, J., and Su, Z., The effect of chitooligosaccharides on oleic acid-induced lipid accumulation in HepG2 cells, Saudi Pharmaceutical Journal (2016).

9. Pan, H., Yang, Q., Huang, G., Ding, C., Cao, P., Huang, L., Xiao, T., Guo, J., and Su, Z., Hypolipidemic effects of chitosan and its derivatives in hyperlipidemic rats induced by a high-fat diet, Food Nutr Res, 60, 31137 (2016). 
10. Zhang, C. M., Yu, S. H., Zhang, L. S., Zhao, Z. Y., and Dong, L. L., Effects of several acetylated chitooligosaccharides on antioxidation, antiglycation and NO generation in erythrocyte, Bioorg Med ChemLett, 24, 4053 (2014).

11. Xing, R., Liu, S., Guo, Z., Yu, H., Wang, P., Li, C., Li, Z., and Li, P., Relevance of molecular weight of chitosan and its derivatives and their antioxidant activities in vitro, Bioorg Med Chem, 13, 1573 (2005).

12. Fang, I. M., Yang, C. H., Yang, C. M., and Chen, M. S., Chitosan oligosaccharides attenuates oxidative-stress related retinal degeneration in rats, PLoS One, 8, e77323 (2013).

13. Liu, X., Sun, Z., Zhang, M., Meng, X., Xia, X., Yuan, W., Xue, F., and Liu, C., Antioxidant and antihyperlipidemic activities of polysaccharides from sea cucumber Apostichopusjaponicus, CarbohydrPolym, 90, 1664 (2012).

14. Sheng, J., and Sun, Y., Antioxidant properties of different molecular weight polysaccharides from Athyriummultidentatum (Doll.) Ching, CarbohydrPolym, 108, 41 (2014).

15. Herraiz, T., and Galisteo, J., Hydroxyl radical reactions and the radical scavenging activity of beta-carboline alkaloids, Food Chem, 172, 640 (2015).

16. Sheng, J., and Sun, Y., Antioxidant properties of different molecular weight polysaccharides from Athyriummultidentatum (Doll.) Ching, CarbohydrPolym, 108, 41 (2014).

17. Liu, X., Sun, Z., Zhang, M., Meng, X., Xia, X., Yuan, W., Xue, F., and Liu, C., Antioxidant and antihyperlipidemic activities of polysaccharides from sea cucumber Apostichopusjaponicus, CarbohydrPolym, 90, 1664 (2012).

18. Liu, W., Wang, J., Zhang, Z., Xu, J., Xie, Z., Slavin, M., and Gao, X., In vitro and in vivo antioxidant activity of a fructan from the roots of Arctiumlappa L, Int J BiolMacromol, 65, 446 (2014).

19. Hill, S. M., Hao, X., Liu, B., and Nystrom, T., Life-span extension by a metacaspase in the yeast Saccharomyces cerevisiae, Science, 344, 1389 (2014).

20. Bulterijs, S., Hull, R. S., Bjork, V. C., and Roy, A. G., It is time to classify biological aging as a disease, Front Genet, 6, 205 (2015).

21. Greer, D. B., Hermanns, M., and Cooper, C. M., Making Lemonade Out of Life's Lemons: A View Into the World of Aging With Parkinson's Disease, J PsychosocNursMent Health Serv, 53, 20 (2015).

22. Miedema, M. D., Lopez, F. L., Blaha, M. J., Virani, S. S., Coresh, J., Ballantyne, C. M., and Folsom, A. R., Implications of the Eighth Joint National Committee Guidelines for the Management of High Blood Pressure for Aging Adults: Atherosclerosis Risk in Communities Study, Hypertension, 66, 474 (2015).

23. Tao, Y., Zhang, H. L., Hu, Y. M., Wan, S., and Su, Z. Q., Preparation of Chitosan and WaterSoluble Chitosan Microspheres via Spray-Drying Method to Lower Blood Lipids in Rats Fed with High-Fat Diets, Int J MolSci, 14, 4174 (2013).

24. Tan, S., Gao, B., Tao, Y., Guo, J., and Su, Z. Q., Antiobese effects of capsaicin-chitosan microsphere (CCMS) in obese rats induced by high fat diet, J Agric Food Chem, 62, 1866 (2014).

25. Feng, T., Du, Y., Li, J., Wei, Y., and Yao, P., Antioxidant activity of half N-acetylated watersoluble chitosan in vitro, European Food Research and Technology, 225, 133 (2007).

26. Huang, L., Chen, J., Cao, P., Pan, H., Ding, C., Xiao, T., Zhang, P., Guo, J., and Su, Z., Anti-obese effect of glucosamine and chitosan oligosaccharide in high-fat diet-induced obese rats, Mar Drugs, 13, 2732 (2015).

27. Qiao, Y., Bai, X., and Du, Y., Chitosan oligosaccharides protect mice from LPS challenge by attenuation of inflammation and oxidative stress, International Immunopharmacology, 11, 121 (2011).

28. Fang, I., Yang, C., and Yang, C., Chitosan oligosaccharides prevented retinal ischemia and reperfusion injury via reduced oxidative stress and inflammation in rats, Experimental Eye Research, 130, 38 (2015). 